\title{
Polyradiculopathies from Schwannomatosis
}

\author{
Yuxia Jia ${ }^{1}$, James A. Kraus ${ }^{2}$, Hasini Reddy ${ }^{2}$, Michael Groff $^{3}$ and Eric T. Wong*,4 \\ ${ }^{1}$ Division of Gerontology, Department of Medicine, Beth Israel Deaconess Medical Center; ${ }^{2}$ Department of Pathology, \\ Beth Israel Deaconess Medical Center; ${ }^{3}$ Division of Neurosurgery, Beth Israel Deaconess Medical Center; ${ }^{4}$ Brain \\ Tumor Center \& Neuro-Oncology Unit, Department of Neurology, Beth Israel Deaconess Medical Center, USA
}

\begin{abstract}
We describe a case of schwannomatosis presenting as radicular pain and numbness in multiple radicular nerve distributions. There were multiple peripheral nerve tumors detected by magnetic resonance imaging (MRI) at the left vestibular nerve, cauda equina, right radial nerve, thoracic paraspinal nerve, and brachial plexi. Several resected tumors have features of schwannomas, including hypercellular Antoni A areas, hypocellular Antoni B areas, Verocay bodies, and hyalinized blood vessels. The specimens are also positive for immunohistochemical staining for INI1 with diffuse nuclear staining. The findings are consistent with sporadic form of schwannomatosis. This case highlights the importance of using MRI and INI1 immunohistochemistry to differentiate familial schwannomatosis, neurofibromatosis 2 (NF2)-associated schwannomatosis, and sporadic schwannomatosis.
\end{abstract}

Keywords: Schwannomatosis, polyradiculopathies, INI1 immunohistochemistry.

\section{LETTER}

A 49-year-old previously healthy Chinese man presented with radicular pain and numbness in the S1 distribution of the left leg. There was no bowel or bladder problem, saddle anesthesia, or weakness. He underwent a gadoliniumenhanced lumbosacral MRI that showed a $1.7 \mathrm{~cm}$ mass compressing the conus of the spinal cord at the level of T12 and L1 (Fig. 1A and 1B, arrows). There was another smaller enhancing mass, measuring $0.7 \mathrm{~cm}$, at L4 (Fig. 1A , arrow). In order to remove mass effect exerted on the conus, he underwent a posterior laminectomy for the surgical removal of the mass at T12-L1. The histological feature of the mass was consistent with schwannoma, World Health Organization grade 1. There were hypercellular Antoni A areas $(*)$, hypocellular Antoni B areas (**), Verocay bodies (arrows), and hyalinized blood vessels (arrowhead) (Fig. 2). Postoperatively, his radicular pain resolved but the S1 numbness remained. He had no family history of schwannoma. But because his past history was notable for removal of another schwannoma from the right superficial paraspinal region, this prompted further staging evaluations to look for schwannomas at other body sites. A head MRI revealed a gadolinium-enhancing mass at the cerebellopontine angle that infiltrates into the left internal auditory canal, measuring $2.7 \mathrm{~cm}$ in diameter, and the neuroradiographic features of this mass was consistent with a vestibular schwannoma (Fig. 3, arrow). There was also a palpable nodule in his right inner arm and compression of this nodule resulted in shooting pain radiating in the radial

*Address correspondence to this author at the Brain Tumor Center \& Neuro-Oncology Unit, Department of Neurology, Beth Israel Deaconess Medical Center, 330 Brookline Avenue, Boston, MA 02215, USA;

Tel: (617) 667-1665; Fax: (617) 667-1664;

E-mail: ewong@bidmc.harvard.edu nerve distribution. An MRI of the brachial plexi showed a $2.0 \mathrm{~cm}$ enhancing mass involving the right radial nerve and multiple smaller gadolinium-enhancing lesions measuring 0.8 to $1.3 \mathrm{~cm}$ causing nodular thickening of the brachial plexi bilaterally (Fig. 4A and 4B, arrows). Because the right radial nerve pain limited his ability to do physical work using his dominant hand, he subsequently underwent surgical removal of this radial nerve mass. The histological feature of this mass was also consistent with schwannoma, and immunohistochemical staining for INI1 demonstrated diffuse nuclear positivity (Fig. 5). Taken together, the presence of multiple schwannomas in peripheral nerves and the diffuse INI1 immunohistochemical staining indicate that our subject has the sporadic form of schwannomatosis.

The differential diagnosis for multiple enhancing nodules in the lumbar thecal sac includes leptomeningeal metastases from systemic malignancies or malignant gliomas, ependymomas, paragangliomas, meningiomatosis, and schwannomatosis. Leptomeningeal metastasis can occur as the first manifestation of cancer in 5 to $10 \%$ of cases but it rarely occurs without manifestations from systemic malignancy $[1,2]$. The intradural metastases may present as linear or nodular enhancements but typically both exist. Ependymomas of the lumbar thecal sac usually have the myxopapillary histology, and myxopapillary ependymoma are invariably solitary at the time of presentation. But drop metastases from supratentorial anaplastic ependymomas are possible. Therefore, patients with multiple enhancing lesions in the lumbar thecal sac need a staging total spine and head MRI to rule out other sites of disease. Paragangliomas arises as a solitary nodule from the filum terminale while meningiomatosis are usually detected in the cranial dura [3]. In our patient, he has multiple schwannomas in a thoracic paraspinal nerve, cauda equina, vestibular nerve, brachial 
A

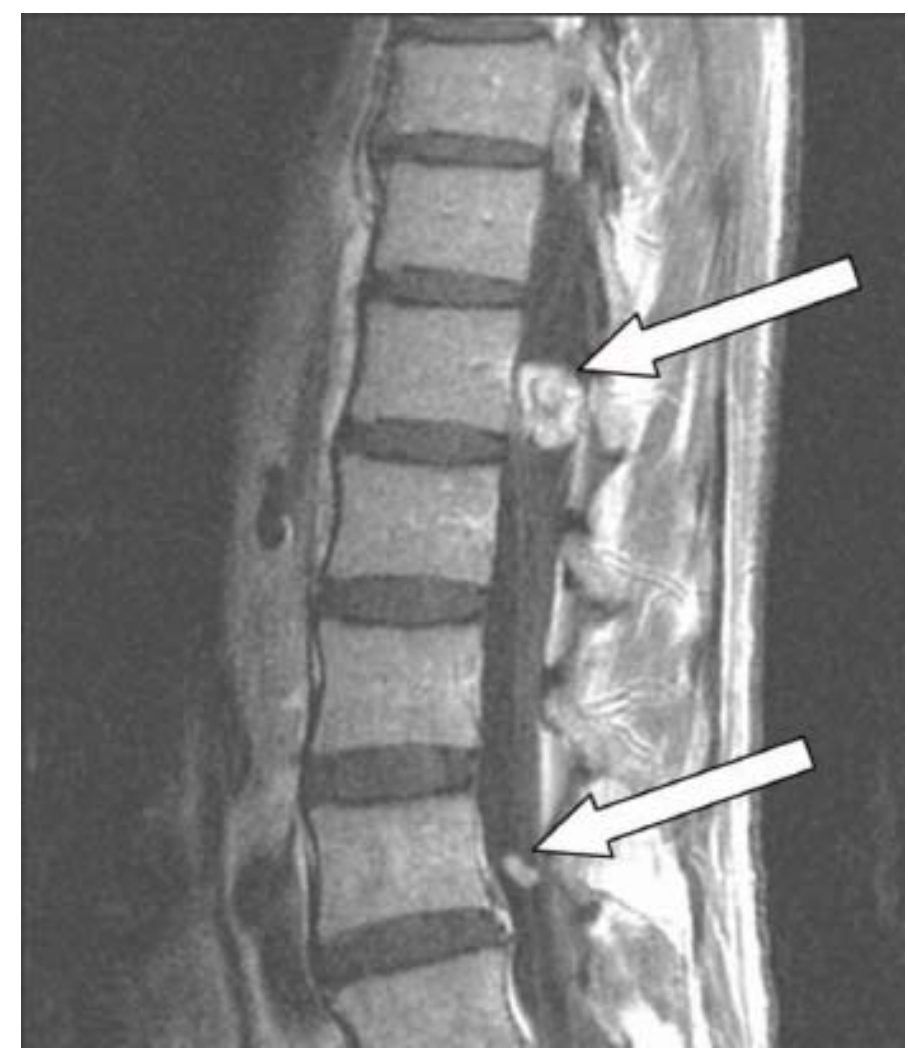

B

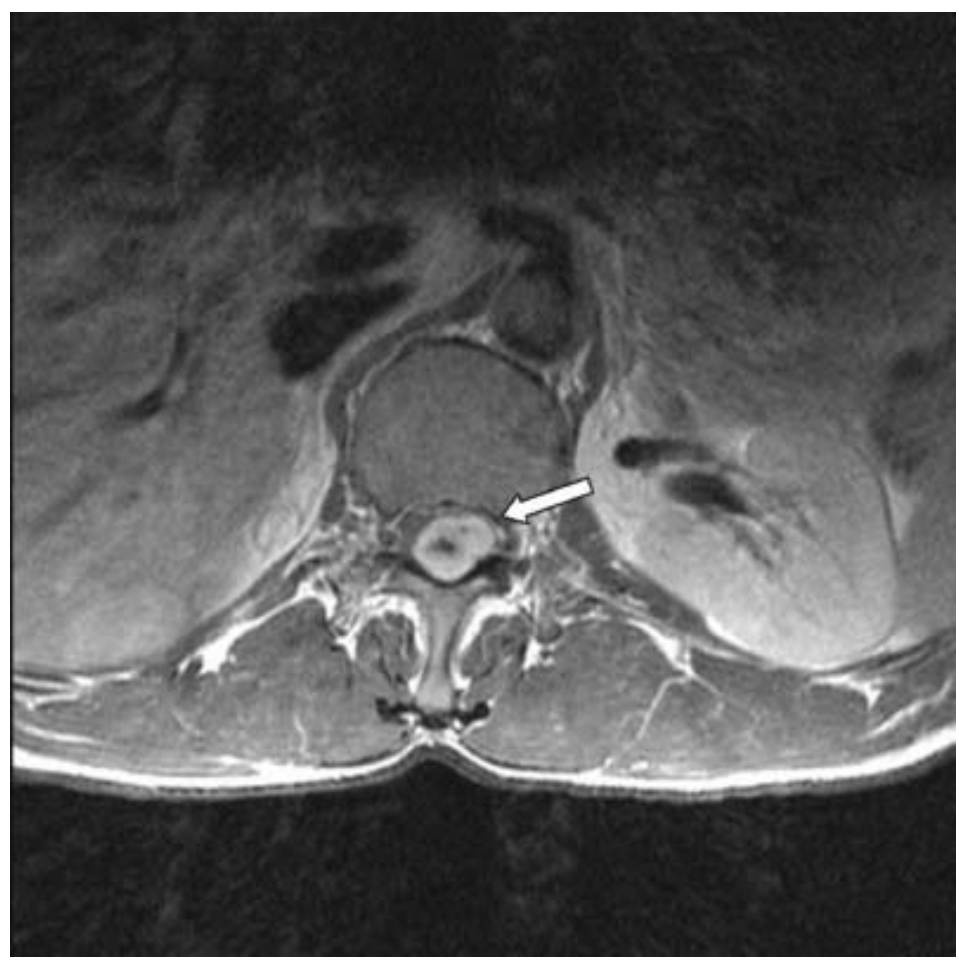

Fig. (1). (A) Sagittal view of lumbosacral spine on post-gadolinium T1-weighted MRI showing a larger mass, measuring $1.7 \mathrm{~cm}$ in diameter, compressing the conus of the spinal cord at the level of T12 and L1 (arrow). A smaller mass, measuring 0.7 in diameter, is also seen at the level of L4 (arrow). (B) Axial view of the larger mass at the level of T12 on post-gadolinium T1-weighted MRI (arrow). 


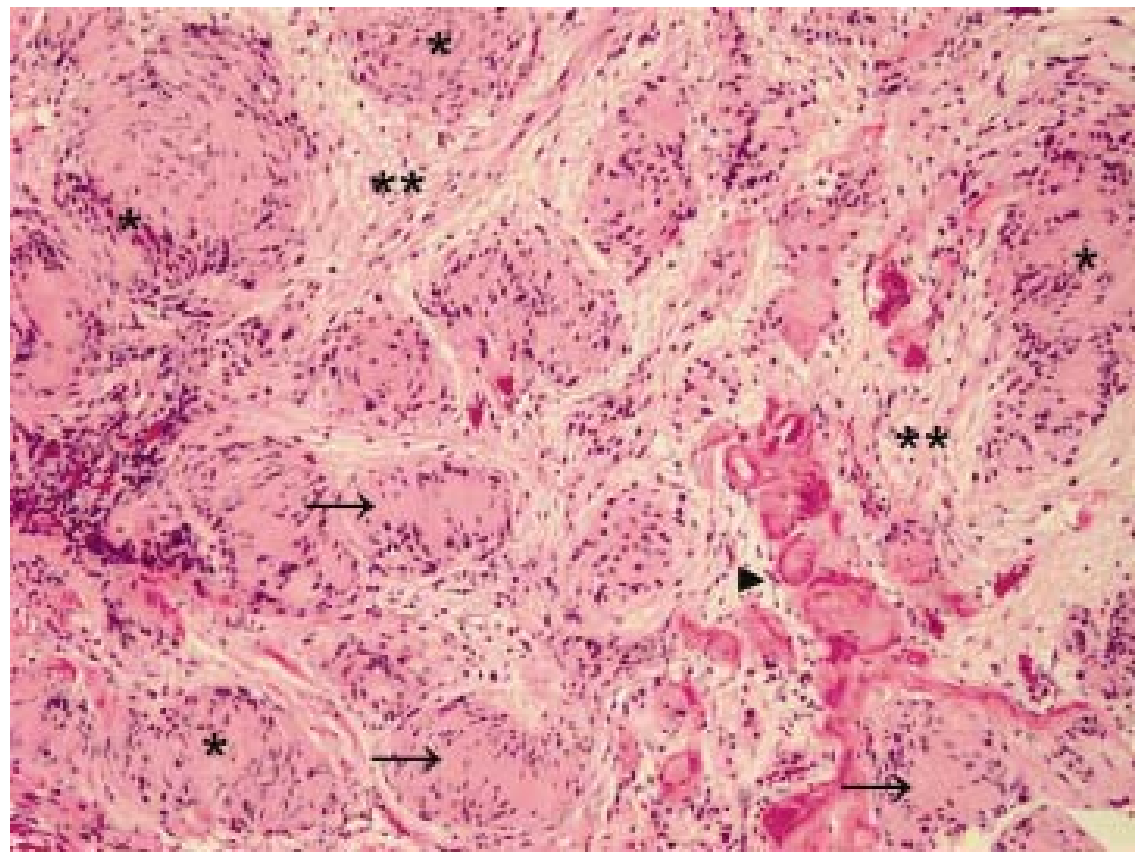

Fig. (2). Hematoxylin and eosin staining of the larger tumor as seen in Figure 1 after surgical removal. Hypercellular Antoni A areas (*), hypocellular Antoni B areas (**), Verocay bodies (arrows), and hyalinized blood vessels (arrowhead) are observed. Original magnification X 100.

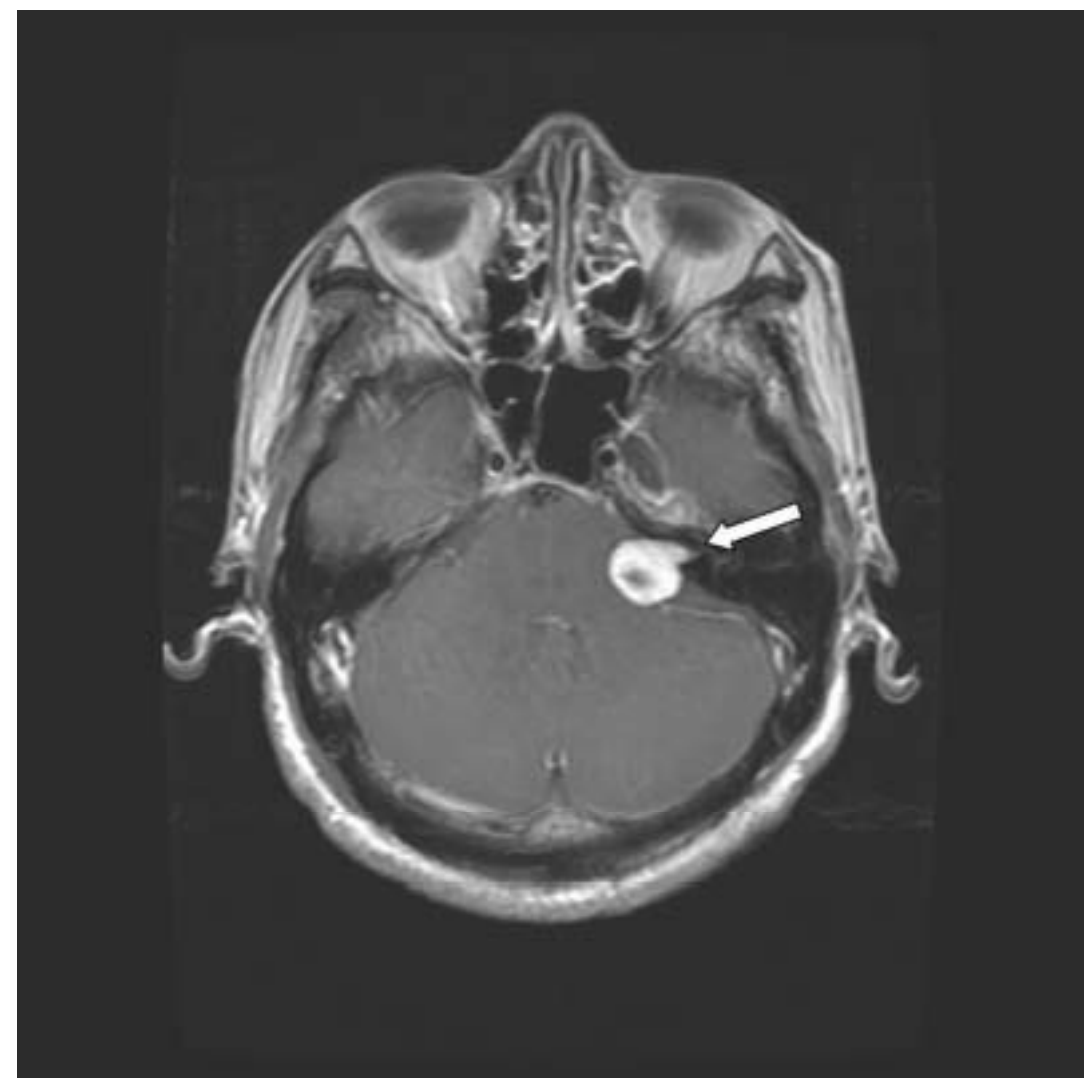

Fig. (3). Gadolinium-enhanced T1-weighted MRI of the head showing a left vestibular schwannoma (arrow).

plexi, and right radial nerve. These findings are consistent with schwannomatosis.

There are three major types of schwannomatosis, which is defined as multiple schwannomas arising from peripheral nerves, including familial schwannomatosis, neurofibromatosis 2 (NF2)-associated schwannomatosis, and sporadic schwannomatosis [4]. The commonly seen sporadic, solitary schwannomas typically arise from the vestibular nerves, 
A

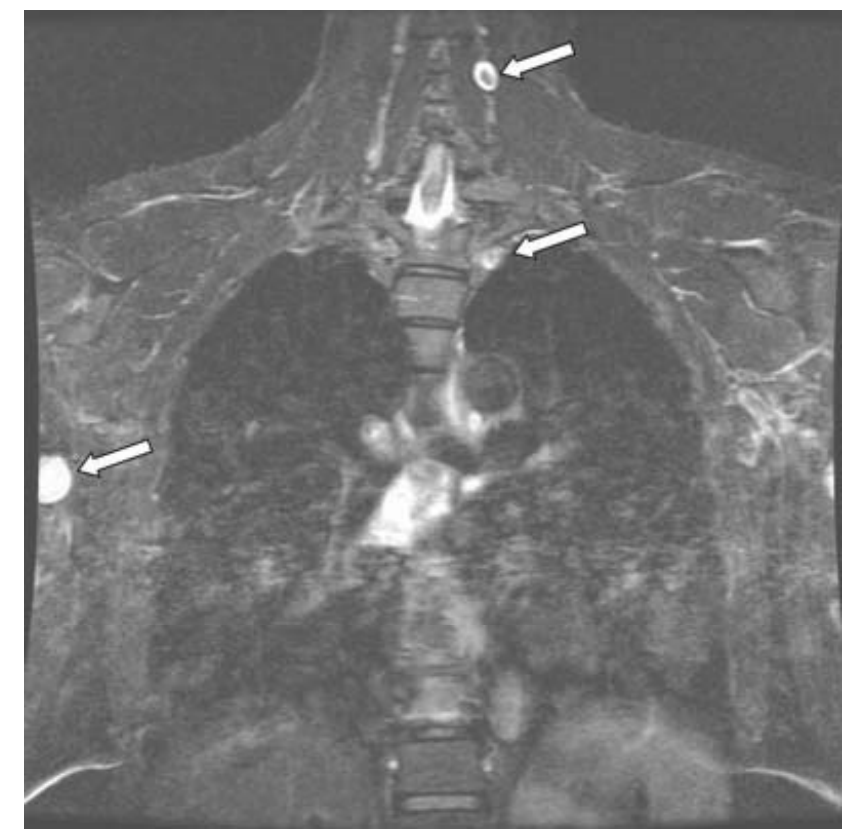

B

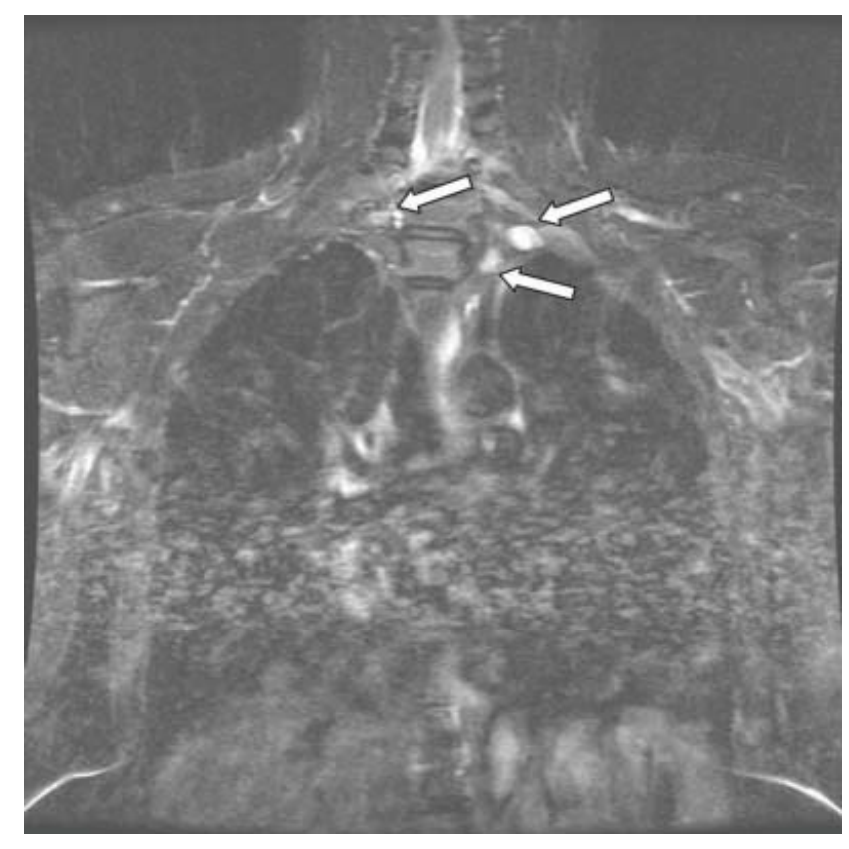

Fig. (4). (A) Coronal view of brachial plexi on post-gadolinium volumetric interpolated brain examination (VIBE) MRI showing two schwannomas (arrows). A third schwannoma is also seen in the right radial nerve (arrows). (B) A separate section of coronal view on post-gadolinium VIBE MRI showing 3 additional schwannomas on the brachial plexi (arrows).

while the presence of bilateral vestibular schwannomas is a hallmark of NF2, which can have manifestations of schwannomas in peripheral nerves as well. The schwannomas in familial and sporadic schwannomatosis are similar, but genetically distinct, to those from NF2. They can be found in intradural locations as in this patient,

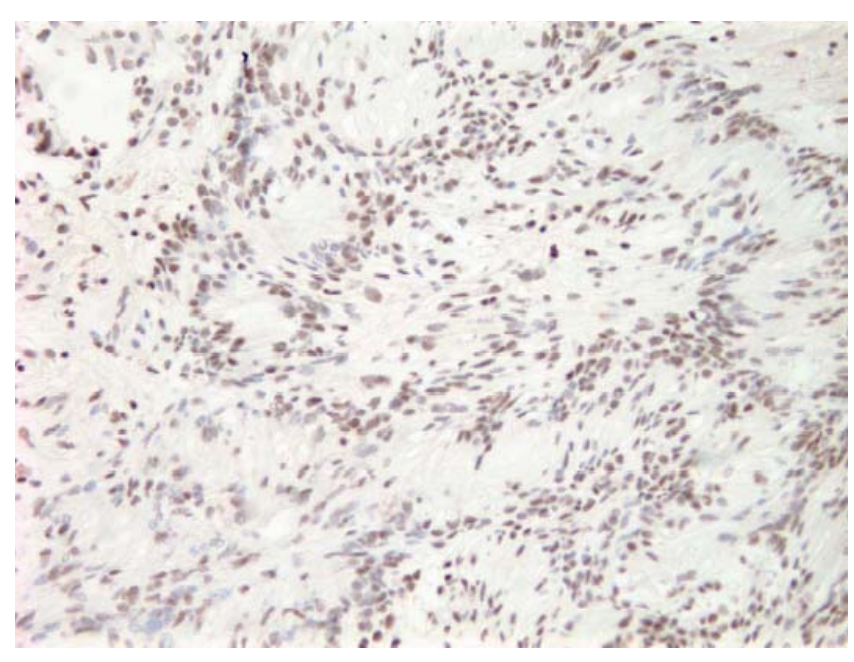

Fig. (5). Immunohistochemical staining for INI1 of the schwannoma removed from the patient's right radial nerve. There is diffuse immunoperoxidase positivity in the nuclei. Original magnification X 200 .

extradurally in lumbosacral or brachial plexi, and the peripheral nerves. Constitutional and somatic mutations of INII/SMARCB1 tumor suppressor gene are associated with familial schwannomatosis. INI1, the protein product of this gene, can be detected in a mosaic pattern (in a mixed pattern of positive and negative immunoreactive nuclei) in $93 \%$ of tumors in familial schwannomatosis, $83 \%$ in NF2 tumors, $55 \%$ in sporadic schwannomatosis, and only $5 \%$ in sporadic, solitary schwannomas [5]. In our patient, the absence of a family history of schwannomas, the lack of bilateral vestibular schwannomas on staging head MRI, and the diffuse, rather than mosaic, staining pattern on INI1 immunohistochemistry all indicate that he had the sporadic form of schwannomatosis. Furthermore, the heterogeneous genetic findings between schwannomatosis and sporadic, solitary schwannoma suggest that these tumors differ in their molecular pathogenesis. It remains to be determined whether or not there are additional genetic defects leading to different phenotypes of familial schwannomatosis, sporadic schwannomatosis, or NF2-associated schwannomatosis.

\section{ACKNOWLEDGEMENT}

This work was supported in part by A Reason To Ride research fund.

\section{ABBREVIATIONS}

INI1

$=$ integrase interactor 1

MRI

$=$ magnetic resonance imaging

$\mathrm{NF} \quad=$ neurofibromatosis

SMARCB1 = SWI/SNF-related matrix-associated actindependent regulator of chromatin subfamily B member 1

\section{REFERENCES}

[1] Wong ET, Joseph JT. Carcinomatous leptomeningeal metastases. J Clin Oncol 2000; 18: 2926-9.

[2] Chamberlain MC. Neoplastic meningitis. J Clin Oncol 2005; 23: 3605-13. 
[3] Wakabayashi K, Kawasaki K, Ono K, et al. Leptomeningeal meningiomatosis with widespread whorl formation. Brain Tumor Pathol 1997; 14: 139-43.

[4] MacCollin M, Chiocca EA, Evans DG, et al. Diagnostic criteria for schwannomatosis. Neurology 2005; 64: 1838-45.
[5] Patil S, Perry A, MacCollin M, et al. Immunohistochemical analysis supports a role for INI1/SMARCB1 in hereditary forms of schwannomas, but not in solitary, sporadic schwannomas. Brain Pathol 2008; 18: 517-9.

Received: August 10, 2010

Revised: October 18, 2010

Accepted: October 25, 2010

(C) Jia et al.; Licensee Bentham Open.

This is an open access article licensed under the terms of the Creative Commons Attribution Non-Commercial License (http://creativecommons.org/licenses/by-nc/3.0/) which permits unrestricted, non-commercial use, distribution and reproduction in any medium, provided the work is properly cited. 